\title{
Rabi-Josephson oscillations and self-trapped dynamics in atomic junctions with two bosonic species
}

\author{
G. Mazzarella ${ }^{1}$, B. Malomed ${ }^{2}$, L. Salasnich ${ }^{1,3}$, M. Salerno ${ }^{4}$, and \\ F. Toigo ${ }^{1}$ \\ ${ }^{1}$ Dipartimento di Fisica "Galileo Galilei" and CNISM, Università di Padova, Via \\ Marzolo 8, 35122 Padova, Italy ${ }^{1}$ \\ ${ }^{2}$ Department of Physical Electronics, Faculty of Engineering, Tel Aviv University, Tel \\ Aviv 69978, Israel \\ ${ }^{3}$ INO-CNR, via G. Sansone 1, 50019 Sesto Fiorentino, Italy \\ ${ }^{4}$ Dipartimento di Fisica "E.R. Caianiello", CNISM and INFN - Gruppo Collegato di \\ Salerno, Università di Salerno, Via Ponte don Melillo, 84084 Fisciano (SA), Italy
}

\begin{abstract}
We investigate the dynamics of two-component Bose-Einstein condensates (BECs), composed of atoms in two distinct hyperfine states, which are linearly coupled by two-photon Raman transitions. The condensate is loaded into a doublewell potential (DWP). A variety of dynamical behaviors, ranging from regular Josephson oscillations, to mixed Rabi-Josephson oscillations and to regimes featuring an increasing complexity, are described in terms of a reduced Hamiltonian system with four degrees of freedoms, which are the numbers of atoms in each component in the left and right potential wells, whose canonically conjugate variables are phases of the corresponding wave functions. Using the system with the four degrees of freedom, we study the dynamics of fractional imbalances of the two bosonic components, and compare the results to direct simulations of the Gross-Pitaevskii equations (GPEs) describing the bosonic mixture. We perform this analysis when the fractional imbalance oscillates around a zero-time averaged value and in the self-trapping regime as well.
\end{abstract}

PACS numbers: 03.75.Ss, $03.75 . \mathrm{Hh}, 64.75 .+\mathrm{g}$ 
Rabi-Josephson oscillations and self-trapped dynamics in atomic junctions with two bosonic species2

\section{Introduction}

The study of Josephson oscillations and self-trapping both with a single bosonic component [1] and in bosonic binary mixtures [2, 3, 4, 5, 6, 7, 9, 10, trapped in doublewell potentials (DWPs) has attracted much interest in the context of the current work on ultracold quantum gases. A specific ramification of this topic corresponds to the situation in which the two components of the mixture are different hyperfine states of the same bosonic atom [2, 3, 6, which may be linearly coupled by an external resonant field [11]. This setting suggests a possibility to study the interplay between Josephson and Rabi oscillations, the latter being induced by the linear interconversion between the components [12, 13]. This subject was considered in several earlier works [14, 15, 17]. In particular, in Ref. [14 the authors analyzed a crossover between the Josephson and Rabi dynamics, using a nonstationary model (with a linearly growing magnetic field), which, in the Josephson limit, was reduced to a system of two degrees of freedom. In Ref. [15], a two-degrees-of-freedom model was used too, with the objective of studying the quasiparticles' spectrum in the symmetry-broken ground state. As suggested in Ref. [16], there exists a possibility to distinguish between the Rabi and Josephson regimes by considering a beam-splitter model based on a nonstationary DWP. An experimental implementation of internal bosonic Josephson junctions with Rubidium spinor BoseEinstein condensate has been recently considered by Zibold et al. [18] in connection with bifurcations occurring at the transition from Rabi to Josephson dynamics.

The natural minimum basis for the analysis of the Rabi-Josephson oscillations in two-component systems in the DWP should include four degrees of freedom [10]. The objective of this work is to analyze physically relevant dynamical regimes within the framework of the minimal system. The predictions will be verified via the comparison to direct simulations of the underlying Gross-Pitaevskii equations (GPEs).

The paper is organized as follows. The model is described in Section II, and the finite-mode approximation is derived in Section III, where we also verify its accuracy by comparison to direct simulations of the underlying GPEs. In Section IV, we report the main results of the work, which demonstrate the interplay between the Josephson and Rabi oscillations, concluding that the oscillations become characterized by an high degree of complexity with the increase of the strength of Rabi coupling. In order to enlarge and complete the analysis presented in Ref. [9], the study of the self-trapping regime is faced as well. In fact, in Section V - within the self-trapping regime - we discuss the accuracy of our model following the same path as in Sec. IV; we comment about the influence of the linear coupling constant on the onset of the self-trapping dynamics. The paper is concluded by Section VI.

\section{The model}

We consider a binary Bose-Einstein condensate of two different species (with index 1 and 2) of repulsively interacting bosons. The condensate is trapped in a DWP, which can be 
produced, for example, by a far off-resonance laser barrier separating each component into two regions, $L$ (left) and $R$ (right). These components may be, for example, two distinct hyperfine states, $\left|F=2, m_{F}=1\right\rangle$ and $\left|F=1, m_{F}=-1\right\rangle$, of ${ }^{87} \mathrm{Rb}$ [2], 3]. Note that since $\left|\Delta m_{F}\right|=2$ one needs two photons to couple the two levels. A weak external magnetic field gives rise to a small difference, $\hbar \omega_{0}$, between the energy levels of these states. Two-photon Raman transitions between the levels, characterized by Rabi frequency $\Omega$, can be induced by a laser beam of frequency $\omega_{d}$, with detuning $\delta \equiv \omega_{d}-\omega_{0}$. Using the rotating-wave approximation (e.g., neglecting high-frequency terms in the atom-field interaction), in the mean-field approximation macroscopic wave functions $\Psi_{n}(\mathbf{r}, t)(n=1,2)$ of the two components of the condensates obey the system of coupled Gross-Pitaevskii equations (GPEs) [12, 19]:

$$
\begin{aligned}
i \hbar \frac{\partial \Psi_{n}}{\partial t} & =-\frac{\hbar^{2}}{2 m} \nabla^{2} \Psi_{n}+\left[V_{\text {trap }}^{(n)}(\mathbf{r})+\frac{(-1)^{n}}{2} \hbar \delta+g_{n}\left|\Psi_{n}\right|^{2}\right. \\
& \left.+g_{12}\left|\Psi_{3-n}\right|^{2}\right] \Psi_{n}+\frac{\Omega}{2} \Psi_{3-n},
\end{aligned}
$$

where $V_{\text {trap }}(\mathbf{r})$ is the trapping potential and $\Psi_{n}(\mathbf{r}, t)$ is subject to the normalization condition,

$$
\int d^{3} \mathbf{r}\left|\Psi_{n}(\mathbf{r}, t)\right|^{2}=N_{n}(t)
$$

with $N_{n}(t)$ the number of bosons of the $n$-th species. Similarly, $m, a_{n}$, and $g_{n}=$ $4 \pi \hbar^{2} a_{n} / m_{n}$ denote, respectively, the atomic mass, $s$-wave scattering length, and intraspecies nonlinearity coefficient of the $n$-th species (the atomic mass is common for both species). The constant accounting for the linear interconversion between the bosonic components is expressed in terms of the respective Rabi frequency, $\Omega$. Finally, $g_{12}=2 \pi \hbar^{2} a_{12} / m$ is the coefficient accounting for the nonlinear interaction between the species, $a_{12}$ being the respective $s$-wave scattering length. Notice that the total number of particles $N_{1}(t)+N_{2}(t)$ is a conserved quantity. In the following, we consider both $g_{n}$ and $g_{12}$ as free parameters, due to the possibility to change the scattering lengths by means of the Feshbach-resonance technique, see, e.g., Ref. [20] and references therein.

Equations (11) can also be derived in a different physical setting, by assuming that the two hyperfine states may be coupled by an external ac magnetic field $B \cos (\omega t)$ of frequency $\omega=\omega_{0}-\delta$. In this case, the linear coupling term in Eq. (11) corresponds to the Rabi frequency $\Omega=\mu \cdot \mathbf{B} / \hbar$, where $\mu$ is the dipole matrix element for the transition between the two hyperfine states [11].

The trapping potential for both components is taken to be the superposition of a strong harmonic confinement in the transverse $(x, y)$ plane and of a DWP in the axial (z) direction, i.e.,

$$
V_{\text {trap }}^{(n)}(\mathbf{r})=\frac{1}{2} m \omega_{n}^{2}\left(x^{2}+y^{2}\right)+V_{\mathrm{DWP}}(z) .
$$

We proceed by writing the Lagrangian associated to the GPEs (11),

$$
L=\int d^{3} \mathbf{r}\left(\left[\sum_{n=1,2} \bar{\Psi}_{n}\left(i \hbar \frac{\partial}{\partial t}+\frac{\hbar^{2}}{2 m_{n}} \nabla^{2}\right) \Psi_{n}\right.\right.
$$


Rabi-Josephson oscillations and self-trapped dynamics in atomic junctions with two bosonic species4

$$
\begin{gathered}
\left.-\left(V_{\text {trap }}^{(n)}(\mathbf{r})\left|\Psi_{n}\right|^{2}+\frac{(-1)^{n} \hbar \delta}{2}\left|\Psi_{n}\right|^{2}+\frac{g_{n}}{2}\left|\Psi_{n}\right|^{4}\right)\right] \\
\left.-(\Omega / 2)\left(\bar{\Psi}_{1} \Psi_{2}+\Psi_{1} \bar{\Psi}_{2}\right)-g_{12}\left|\Psi_{1}\right|^{2}\left|\Psi_{2}\right|^{2}\right)
\end{gathered}
$$

where $\bar{\Psi}_{n}$ stands for the complex conjugate of $\Psi_{n}$. To derive, at first, the $1 \mathrm{D}$ approximation, we adopt the usual ansatz

$$
\Psi_{n}(x, y, z, t)=\frac{1}{\sqrt{\pi} a_{\perp, n}} \exp \left(-\frac{x^{2}+y^{2}}{2 a_{\perp, n}^{2}}\right) f_{n}(z, t),
$$

where $a_{\perp, n}=\sqrt{\hbar /\left(m \omega_{n}\right)}$ are the respective transverse-confinement radii, with the 1D wave functions $f_{n}(z, t)$ obeying normalization conditions $\int d z\left|f_{n}(z, t)\right|^{2}=N_{n}(t)$. Note that the factorized ansatz (5) is valid under the strong transverse confinement, viz., when $g_{n}\left|f_{n}\right|^{2} / 4 \pi a_{\perp, n}^{2} \ll \hbar \omega_{n}$ [21]. By inserting the ansatz (5]) into the Lagrangian (4) and performing the integration in the transverse plane, we derive the effective Lagrangian for the $1 \mathrm{D}$ wave functions:

$$
\begin{aligned}
L_{1 \mathrm{D}} & =\int d z\left(\left[\sum_{n=1,2} \bar{f}_{n}\left(i \hbar \frac{\partial}{\partial t}+\frac{\hbar^{2}}{2 m} \frac{\partial^{2}}{\partial z^{2}}\right) f_{n}\right.\right. \\
& \left.-\left[\epsilon_{n}+V_{\mathrm{DWP}}(z)+\frac{(-1)^{n} \hbar \delta}{2}\right]\left|f_{n}\right|^{2}-\frac{\tilde{g}_{n}}{2}\left|f_{n}\right|^{4}\right] \\
& \left.-(\tilde{\Omega} / 2)\left(\bar{f}_{1} f_{2}+f_{1} \bar{f}_{2}\right)-\tilde{g}_{12}\left|f_{1}\right|^{2}\left|f_{2}\right|^{2}\right),
\end{aligned}
$$

where the following constants are introduced:

$$
\begin{aligned}
& \epsilon_{n}=(1 / 2)\left[\hbar^{2} /\left(m a_{n, \perp}^{2}\right)+m \omega_{n}^{2} a_{n, \perp}^{2}\right], \\
& \tilde{g}_{n}=g_{n} /\left(2 \pi a_{\perp, n}^{2}\right), \\
& \tilde{\Omega}=2 a_{\perp, 1} a_{\perp, 2}\left(a_{\perp, 1}^{2}+a_{\perp, 2}^{2}\right)^{-1} \Omega,
\end{aligned}
$$

and

$$
\tilde{g}_{12}=\left(g_{12} / \pi\right)\left(a_{\perp, 1}^{2}+a_{\perp, 2}^{2}\right)^{-1} .
$$

By varying $L_{1 \mathrm{D}}$ with respect to $\bar{f}_{n}$, we derive the effective 1D GPEs,

$$
\begin{aligned}
i \hbar \frac{\partial f_{n}}{\partial t} & =-\frac{\hbar^{2}}{2 m_{n}} \frac{\partial^{2} f_{n}}{\partial z^{2}}+\left[\epsilon_{n}+V_{\mathrm{DWP}}(z)+\frac{(-1)^{n} \hbar \delta}{2}\right. \\
& \left.+\tilde{g}_{n}\left|f_{n}\right|^{2}+\tilde{g}_{12}\left|f_{3-m}\right|^{2}\right] f_{n}+(\tilde{\Omega} / 2) f_{3-m} .
\end{aligned}
$$

\section{The finite-mode system}

To approximate the dynamics by a finite-mode approximation, we make use of the two-mode decomposition for each wave function, as originally introduced in Ref. [22]:

$$
f_{n}(z, t)=\psi_{n}^{L}(t) \phi_{n}^{L}(z)+\psi_{n}^{R}(t) \phi_{n}^{R}(z) .
$$


Rabi-Josephson oscillations and self-trapped dynamics in atomic junctions with two bosonic species5

The orthonormal real functions $\phi_{n}^{\alpha}(z)$ are constructed according to the same path as in Ref. [7] and as commented below. These functions are localized in the left and in the right wells, respectively $(\alpha=L, R)$ [7], and

$$
\psi_{n}^{\alpha}(t) \equiv \sqrt{N_{n}^{\alpha}(t)} e^{i \theta_{n}^{\alpha}(t)},
$$

with the total number of particles in the $n$-th species being $N_{n}^{L}(t)+N_{n}^{R}(t)=\left|\psi_{n}^{L}(t)\right|^{2}+$ $\left|\psi_{n}^{R}(t)\right|^{2} \equiv N_{n}(t)$. As described in the Appendix, we derive explicit evolution equations for the temporal evolution of the fractional imbalances, $z_{n}=\left(N_{n}^{L}-N_{n}^{R}\right) / N_{n}$ and intraspecies relative phases, $\theta_{n}=\theta_{n}^{R}-\theta_{n}^{L}$ :

$$
\begin{aligned}
\dot{z}_{n}= & -\frac{2\left(K_{n}-K_{c, n} N_{n}\right)}{\hbar} \sqrt{1-z_{n}^{2}} \sin \theta_{n}+\frac{V_{n} N_{n}}{2 \hbar}\left(1-z_{n}^{2}\right) \sin 2 \theta_{n} \\
+ & \frac{2}{\hbar}\left(V_{12} \sqrt{1-z_{3-n}^{2}} \cos \theta_{3-n}+K_{c, 12}\right) N_{3-n} \sqrt{1-z_{n}^{2}} \sin \theta_{n} \\
& \mp \frac{\tilde{\Omega}}{2 \hbar} \sqrt{N_{1} N_{2}}\left(\sqrt{\left(1+z_{1}\right)\left(1+z_{2}\right)} \sin \gamma_{L}-\sqrt{\left(1-z_{1}\right)\left(1-z_{2}\right)} \sin \gamma_{R}\right), \\
\dot{\theta}_{n}= & \frac{U_{n}-V_{n}}{\hbar} N_{n} z_{n}+\frac{2\left(K_{n}-K_{c, n} N_{n}\right)}{\hbar} \frac{z_{n} \cos \theta_{n}}{\sqrt{1-z_{n}^{2}}} \\
- & \frac{V_{n} N_{n}}{2 \hbar} z_{n} \cos 2 \theta_{n}+\frac{U_{12}-V_{12}}{\hbar} N_{3-n} z_{3-n} \\
- & \frac{2}{\hbar}\left[V_{12} \sqrt{1-z_{3-n}^{2}} \cos \theta_{3-n}+K_{c, 12}\right] N_{3-n} \frac{z_{n} \cos \theta_{n}}{\sqrt{1-z_{n}^{2}}} \\
+ & \frac{\tilde{\Omega}}{2 \hbar} \sqrt{\frac{N_{3-n}}{N_{n}}}\left(\sqrt{\frac{1+z_{3-n}}{1+z_{n}}} \cos \gamma_{L}-\sqrt{\left.\frac{1-z_{3-n}}{1-z_{n}} \cos \gamma_{R}\right) .}\right.
\end{aligned}
$$

Here the signs plus and minus pertain to $n=1$ and 2 , respectively. The total numbers of particles $N_{n}$ of each component and the respective phases, $\gamma_{\alpha}=\theta_{1}^{\alpha}-\theta_{2}^{\alpha}($ recall $\alpha=L, R$ ) evolve according to:

$$
\begin{aligned}
& \dot{N}_{n}= \pm\left(-\frac{\tilde{\Omega}}{2 \hbar} \sqrt{N_{1} N_{2}}\right)\left(\sqrt{\left(1+z_{1}\right)\left(1+z_{2}\right)} \sin \gamma_{L}+\sqrt{\left(1-z_{1}\right)\left(1-z_{2}\right)} \sin \gamma_{R}\right), \\
& \dot{\gamma}_{L}=\frac{1}{2 \hbar}\left(N_{1}\left(U_{12}-U\right)\left(1+z_{1}\right)-N_{2}\left(U_{12}-U\right)\left(1+z_{2}\right)+\Delta E\right) \\
+ & \frac{1}{\hbar}\left(K_{1} \sqrt{\frac{1-z_{1}}{1+z_{1}}} \cos \theta_{1}-K_{2} \sqrt{\frac{1-z_{2}}{1+z_{2}}} \cos \theta_{2}\right) \\
- & \frac{\tilde{\Omega}}{2 \hbar \sqrt{N_{1} N_{2}}}\left(\frac{N_{2}\left(1+z_{2}\right)-N_{1}\left(1+z_{1}\right)}{\sqrt{\left(1+z_{1}\right)\left(1+z_{2}\right)}}\right) \cos \gamma_{L} \\
+ & \frac{K_{c, 12}}{\hbar}\left[N_{1} \sqrt{1-z_{1}^{2}} \cos \theta_{1}-N_{2} \sqrt{1-z_{2}^{2}} \cos \theta_{2}\right]- \\
& \frac{V}{2 \hbar}\left[N_{1}\left(1-z_{1}\right)\left(2+\cos 2 \theta_{1}\right)-N_{2}\left(1-z_{2}\right)\left(2+\cos 2 \theta_{2}\right)\right]- \\
& \frac{1}{\hbar}\left[K_{c, 1} N_{1}\left(2+z_{1}\right)+K_{c, 12} N_{2}+V_{12} N_{2} \sqrt{1-z_{2}^{2}} \cos \theta_{2}\right] \sqrt{\frac{1-z_{1}}{1+z_{1}}} \cos \theta_{1}+ \\
& \frac{1}{\hbar}\left[K_{c, 2} N_{2}\left(2+z_{2}\right)+K_{c, 12} N_{1}\right.
\end{aligned}
$$


Rabi-Josephson oscillations and self-trapped dynamics in atomic junctions with two bosonic species6

$$
\begin{aligned}
+ & \left.V_{12} N_{1} \sqrt{1-z_{1}^{2}} \cos \theta_{1}\right] \sqrt{\frac{1-z_{2}}{1+z_{2}}} \cos \theta_{2}+\frac{V_{12}}{2}\left(N_{1}\left(1-z_{1}\right)-N_{2}\left(1-z_{2}\right)\right), \\
& \dot{\gamma}_{R}=\frac{1}{2 \hbar}\left(N_{1}\left(U_{12}-U\right)\left(1-z_{1}\right)-N_{2}\left(U_{12}-U\right)\left(1-z_{2}\right)+\Delta E\right) \\
+ & \frac{1}{\hbar}\left(K_{1} \sqrt{\frac{1+z_{1}}{1-z_{1}}} \cos \theta_{1}-K_{2} \sqrt{\frac{1+z_{2}}{1-z_{2}}} \cos \theta_{2}\right)- \\
& \frac{\tilde{\Omega}}{\hbar \sqrt{N_{1} N_{2}}}\left(\frac{N_{2}\left(1-z_{2}\right)-N_{1}\left(1-z_{1}\right)}{\sqrt{\left(1-z_{1}\right)\left(1-z_{2}\right)}}\right) \cos \gamma_{R} \\
+ & \frac{K_{c, 12}}{\hbar}\left[N_{1} \sqrt{1-z_{1}^{2}} \cos \theta_{1}-N_{2} \sqrt{1-z_{2}^{2}} \cos \theta_{2}\right]- \\
& \frac{V}{2 \hbar}\left[N_{1}\left(1+z_{1}\right)\left(2+\cos 2 \theta_{1}\right)-N_{2}\left(1+z_{2}\right)\left(2+\cos 2 \theta_{2}\right)\right]- \\
& \frac{1}{\hbar}\left[K_{c, 1} N_{1}\left(2-z_{1}\right)+K_{c, 12} N_{2}+V_{12} N_{2} \sqrt{1-z_{2}^{2}} \cos \theta_{2}\right] \sqrt{\frac{1+z_{1}}{1-z_{1}}} \cos \theta_{1}+ \\
& \frac{1}{\hbar}\left[K_{c, 2} N_{2}\left(2-z_{2}\right)+K_{c, 12} N_{1}\right. \\
+ & \left.V_{12} N_{1} \sqrt{1-z_{1}^{2}} \cos \theta_{1}\right] \sqrt{\frac{1+z_{2}}{1-z_{2}}} \cos \theta_{2}+\frac{V_{12}}{2}\left(N_{1}\left(1+z_{1}\right)-N_{2}\left(1+z_{2}\right)\right)
\end{aligned}
$$

where $\Delta E \equiv E_{2}-E_{1}=\hbar \delta$. The latter relation follows from the normalization of functions $\phi_{\alpha}$ and the first equation from Eq. (20). Obviously, in the absence of Rabi coupling, i.e. $\tilde{\Omega}=0$, the total number of particles is conserved in each component. In that case, Eqs. (13) reduce to the equations of motion for coupled pendula derived in Ref. [7]. We observe that due to the nonlinearity associated to the intra- and inter-species interactions, the system is nonintegrable also when $\tilde{\Omega}=0$. On the other hand, in the absence of the Josephson coupling, i.e., when $K_{n}=0$, the dynamics will be characterized by independent Rabi oscillations in each well; when the Josephson coupling is finite, i.e., $K_{n} \neq 0$, and much smaller than $\tilde{\Omega}$, the aforementioned single-well independent Rabi oscillations will be weakly coupled by the Josephson tunneling. Note that both when $K_{n}=0$ and $K_{n} \neq 0$, the Rabi interconversions will be deformed by nonlinear effects due to the intra- and the inter-species interactions.

In our calculations the axial double-well potential is given by

$$
V_{D W P}(z)=-U_{0}\left[\operatorname{sech}^{2}\left(\frac{z+z_{0}}{\mathcal{A}}\right)+\operatorname{sech}^{2}\left(\frac{z-z_{0}}{\mathcal{A}}\right)\right]
$$

with

$$
U_{0}=\hbar \omega_{\perp}\left[1+\operatorname{sech}^{2}\left(\frac{2 z_{0}}{\mathcal{A}}\right)\right]^{-1}
$$

that is the combination of two Pöschl-Teller (PT) potentials separated by a potential barrier the height of which may be changed by changing $\mathcal{A}$, and centered around the points $-z_{0}$ and $z_{0}$. The the wave functions of the eigenvalues problem in the presence of the only potential $V_{\alpha}(z)(\alpha=L, R)$ are exactly known. In particular, the wave function 
Rabi-Josephson oscillations and self-trapped dynamics in atomic junctions with two bosonic species7

of the ground state is 23

$$
\begin{aligned}
& \phi_{n}^{\alpha, P T}(z)=B\left[1-\operatorname{Tanh}^{2}\left(\frac{z \mp z_{0}}{\mathcal{A}}\right)\right]^{C_{n} / 2} \\
& C_{n}=-\frac{1}{2}+\sqrt{\frac{2 m U_{0} \mathcal{A}^{2}}{\hbar^{2}}+\frac{1}{4}} .
\end{aligned}
$$

If $\alpha=L(R)$, the function $\phi_{n}^{\alpha, P T}(z)$ is centered around $-z_{0}\left(+z_{0}\right)$, and it is the ground state wave function of the PT potential centered around the point $-z_{0}\left(z_{0}\right)$. In Eq. (17) $B$, equal for both sides, ensures the normalization of the wave function. The above functions $\phi_{n}^{L}(z)$ and $\phi_{n}^{R}(z)$. Then, proceeding from the functions (17), $\phi_{L}(z)$ and $\phi_{R}(z)$ can be determined following the same perturbative approach as in [7], where it is shown that, under certain hypothesis, the aforementioned functions can be written in terms of $\phi_{n}^{L, P T}(z)$ and $\phi_{n}^{R, P T}(z)$ given by (17). We get [7]

$$
\begin{aligned}
& \phi_{n}^{L}(z)=\frac{1}{2}\left[\left(\frac{1}{\sqrt{1+s}}+\frac{1}{\sqrt{1-s}}\right) \phi_{n}^{L, P T}(z)+\left(\frac{1}{\sqrt{1+s}}-\frac{1}{\sqrt{1-s}}\right) \phi_{n}^{R, P T}(z)\right] \\
& \phi_{n}^{R}(z)=\frac{1}{2}\left[\left(\frac{1}{\sqrt{1+s}}-\frac{1}{\sqrt{1-s}}\right) \phi_{n}^{L, P T}(z)+\left(\frac{1}{\sqrt{1+s}}+\frac{1}{\sqrt{1-s}}\right) \phi_{n}^{R, P T}(z)\right],
\end{aligned}
$$

where $s=\int_{-\infty}^{+\infty} d z \phi_{n}^{L, P T}(z) \phi_{n}^{R, P T}(z)$.

The relations between the macroscopic parameters involved at right hand sides of Eqs. (13)-(14) and the microscopic parameters of the problem are reported in the Eq. (20) of the Appendix. We observe, moreover, that to make the system fully symmetric we also assume that $K_{1}=K_{2} \equiv K, U_{1}=U_{2} \equiv U, K_{c, 1}=K_{c, 2} \equiv K_{c}$, and $V_{1}=V_{2} \equiv V$.

Obviously, in the absence of Rabi coupling, i.e., $\tilde{\Omega}=0$, the total number of particles is conserved in each component. In that case, Eqs. (13) reduce to the equations of motion for coupled pendula derived in Ref. [7]. Note, moreover, that in our calculations we have assumed that the both the components feel the same harmonic potential so that $\omega_{1}=\omega_{2} \equiv \omega_{\perp}$.

To verify the reliability of the finite-mode approximation which leads to the ODEs (13) and (14) )-(14), we compare the evolution of fractional imbalances $z_{n}$, as predicted by this system, with results of direct numerical simulations of the 1D GPEs (10) both when the fractional imbalances $z_{n}(t)$ oscillate around a zero-time averaged value and when the time-averaged value of $\left\langle z_{n}(t)\right\rangle \neq 0$, that is the self-trapping regime. For the oscillations characterized by $\left\langle z_{n}(t)\right\rangle=0$, the results of the comparison are presented in Fig. 1. This figure shows a good agreement, especially when the Rabi coupling, $\tilde{\Omega}$, is small enough. At larger values of $\tilde{\Omega}$, the finite-mode approximation demonstrates a deviation, which accumulates at sufficiently long times.

In Fig. 2, we report the above comparison between GPEs and ODEs when the fractional imbalances are both self-trapped. Also in this case the distance between the predictions from the two approaches increases for long times when $\tilde{\Omega}$ is big enough. It is worth to observe that the authors of Ref. [9] have performed the comparison between the predictions of GPEs and those ones ODEs deriving from the two-mode approximation only when the fractional imbalances oscillate around zero. Julia-Diaz and co-workers, 
Rabi-Josephson oscillations and self-trapped dynamics in atomic junctions with two bosonic species8
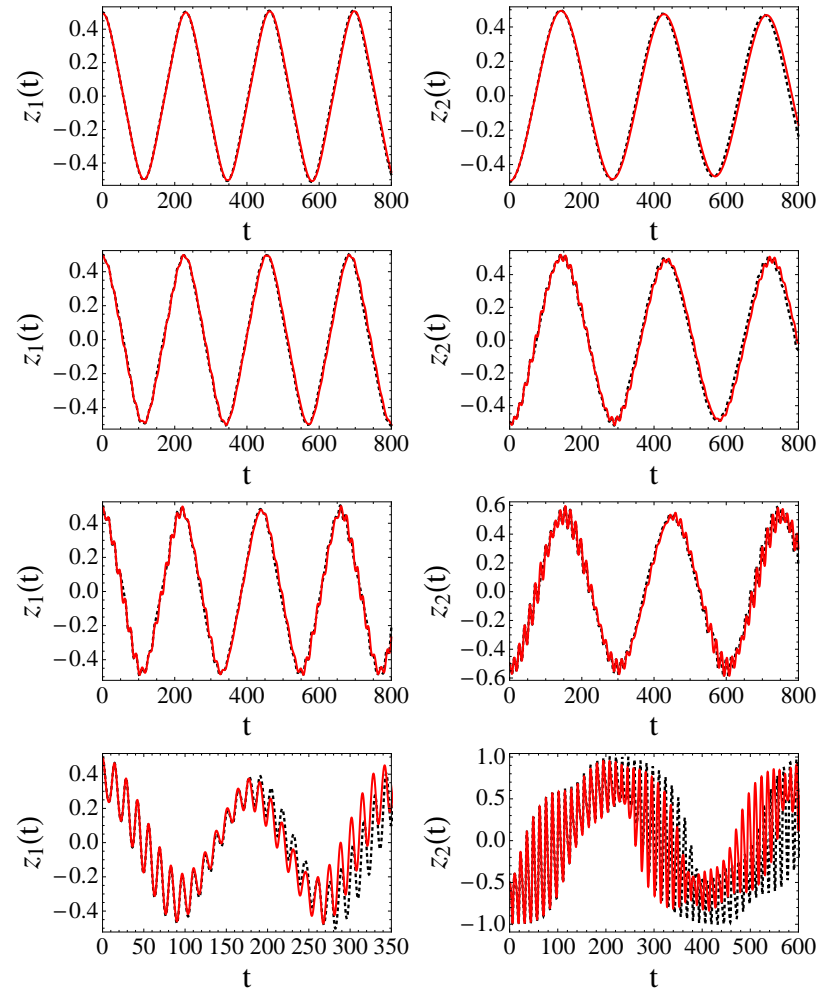

Figure 1. Fractional imbalances $z_{1}$ and $z_{2}$ of the two components vs time $t$ in the Josephson regime. Solid lines: 1D GPE, Eqs. (10). Dotted lines: finite-mode equations (13) - (14). Upper panels: $\tilde{\Omega}=0$ (no Rabi coupling); central panels: $\tilde{\Omega}=K$; third row: $\tilde{\Omega}=3 K$; lower panels: $\tilde{\Omega}=20 K$. The parameters of the double-well potential (15) are $\mathcal{A}=1$ and $z_{0}=3 . K_{1}=K_{2} \equiv K=4.955 \times 10^{-3}, U_{1}=U_{2} \equiv U=0.1 K$, and $U_{12}=-0.01 U$. Other parameters: $K_{c}=-3.684 \times 10^{-6}, V=2.268 \times 10^{-7}$, $K_{c, 12}=-0.01 K_{c}, V_{12}=-0.005 \mathrm{~V} ; E_{2}-E_{1}=-0.39$, as in Ref. [12. Initial conditions: $N_{1}(0)=200, N_{2}(0)=100 z_{1}(0)=0.5=-z_{2}(0), \theta_{n}(0)=0, \gamma_{\alpha}(0)=0$. Time in units of $\omega_{\perp}^{-1}$, lengths in units of $a_{\perp}$, and energies in units of $\hbar \omega_{\perp}$.

moreover, have integrated the aforementioned ODEs under the assumptions of small imbalances, and small intra- and inter-species phase differences [9].

\section{Josephson and Rabi oscillations mixing and the self-trapped dynamics}

One of the central theme of this work is the interplay of the Josephson oscillations and Rabi oscillations. We start with the situation in which only the Josephson coupling is present, i.e., $\tilde{\Omega}=0$, see the upper panels of Fig. 1 and of Figs. 3, 4. In the other panels of these figures we report the behavior of the system in the presence of finite values of $\tilde{\Omega}$. One can see that, the greater is the Rabi coupling between the two species (hyperfine atomic states), the greater is the dynamical complexity exhibited by the system, as observed, especially, in the lower panels of Figs. 1, 3 and 4. Moreover, as shown in the lower panels of Fig. 1, when $\tilde{\Omega}=20 K$, the behaviors of $z_{1}(t)$ and $z_{2}(t)$ are strongly asymmetric with respect to each other; this asymmetry is absent when $E_{2}-E_{1}=0$. 
Rabi-Josephson oscillations and self-trapped dynamics in atomic junctions with two bosonic species9
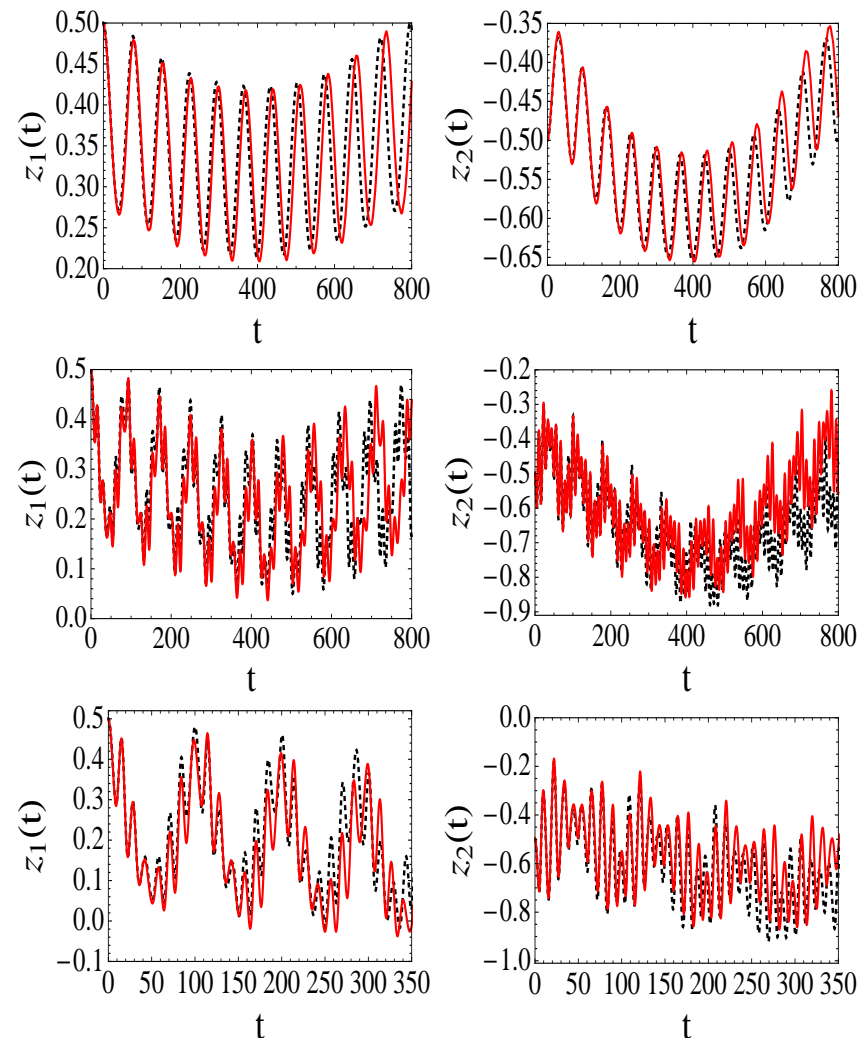

Figure 2. Fractional imbalances $z_{1}$ and $z_{2}$ of the two components vs time $t$ in the selftrapping regime. Solid lines: 1D GPE, Eqs. (10). Dotted lines: finite-mode equations (13) - Upper panels: $\tilde{\Omega}=0$ (no Rabi coupling); central panels: $\tilde{\Omega}=5 K$; lower panels: $\tilde{\Omega}=10 \mathrm{~K}$. We choose $K_{1}=K_{2} \equiv K=4.955 \times 10^{-3}, U_{1}=U_{2} \equiv U=0.1 K$, and $U_{12}=-2 U, K_{c, 12}=-2 K_{c}, V_{12}=-V$. Other parameters, initial conditions and units as in Fig. 1

In Fig. 3 we plot the dynamics of the finite-dimensional system in the planes of fractional imbalances $z_{n}(t)$ and phases $\theta_{n}(t)$, using the trapping and input parameters of Fig. 1. The figure shows the motion is fully periodic for $\tilde{\Omega}=0$, than it becomes quasiperiodic and finally aperiodic by increasing the value of $\tilde{\Omega}$. To quantify the increasing of complexity with the growth of $\tilde{\Omega}$, in Fig. 4 we show the power spectrum of the oscillations, represented by the absolute value, $\left|z_{n}(\omega)\right|$, of the Fourier transform of $z_{n}(t)$, denoting by $\bar{\omega}_{1}$ the frequency associated to the maximum of $\left|z_{1}(\omega)\right|$, and by $\bar{\omega}_{2}$ the one associated to the maximum of $\left|z_{2}(\omega)\right|$. The frequencies $\bar{\omega}_{n}$ are the fundamental frequencies of $z_{n}(t)$, i.e. the frequencies of the carrier waves of $z_{n}(t)$. Let us focus on Table 1 obtained with $N_{1}(0)=200>N_{2}(0)=100$. From the data there reported, we see that - whatever is the value of the Rabi coupling $\tilde{\Omega}-\bar{\omega}_{1}>\bar{\omega}_{2}$. Then the multiparticle tunneling period associated to $\bar{\omega}_{1}, \bar{T}_{1}$, given by $2 \pi / \bar{\omega}_{1}$, is smaller than the one associated to $\bar{\omega}_{2}, \bar{T}_{2}$, given by $2 \pi / \bar{\omega}_{2}$. This is due to the fact that - within a mechanical analogy - the ODEs equations (13)-(14) describes two coupled pendula. At least in the presence of sufficiently weak inter-species interactions, the mass of each pendulum is related to the inverse of the particles number $N_{n}$ of the $n$th species, as discussed for 
Rabi-Josephson oscillations and self-trapped dynamics in atomic junctions with two bosonic species 10
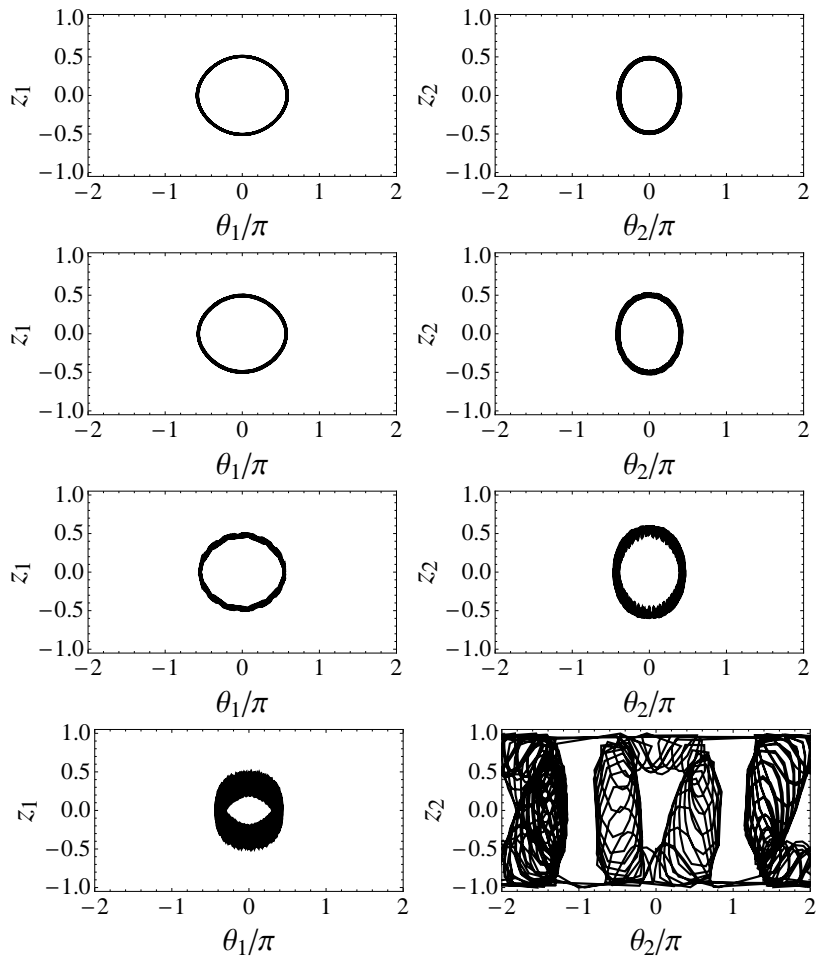

Figure 3. Josephson regime. The dynamics of the finite-dimensional system in the planes of fractional imbalances $z_{n}(t)$ and phases $\theta_{n}(t)$. The parameters, initial conditions, and units as in Fig. 1.

a single component in Ref. [1. From the Table 1, it is possible infer as well that the greater is $\tilde{\Omega}$ the greater is the ratio $\bar{T}_{2} / \bar{T}_{1}$. On the other hand, if we keep fixed the Rabi coupling and increase $N_{1}(0)$ with respect to $N_{2}(0)$, the period $\bar{T}_{1}$ will be smaller and smaller if compared with $\bar{T}_{2}$. Moreover, from Table 1 again, we see that the relative changes $r_{n}$ of $\bar{\omega}_{n}$ with respect to their values at $\tilde{\Omega}=0$ increase by increasing $\tilde{\Omega}$.

From Fig. 4 it is possible to gain physical insight in the dynamics of the system, especially for large values of the Rabi coupling $\tilde{\Omega}$. We can see that when $\tilde{\Omega}$ is sufficiently large, $\left|z_{n}(\omega)\right|$ exhibits a multi-peak structure related to the appearance of frequencies different from the fundamental one. This reflects in an increasing number of harmonics involved in $z_{n}(t)$ (see, in particular, the lower panels of Fig. 1 where $\tilde{\Omega}=20 K$ ) and, accordingly, in an increasing degree of complexity. By analyzing Fig. 4 again, one can conclude that the power spectrum approaches that of random oscillations as $\tilde{\Omega}$ increases. This, immediately, reflects on a quite complicated dynamics when we focus on the plane $z_{n}(t)-\theta_{n}(t)$ (see, in particular, the lower panels in Fig. 3 where $\tilde{\Omega}=20 K$ ). Finally, note that the complexity pertaining to high values of $\tilde{\Omega}$ increases if the dynamics is observed on sufficiently long time scales as shown in the lower panels of Fig. 3 and Fig. 4 
Rabi-Josephson oscillations and self-trapped dynamics in atomic junctions with two bosonic species11
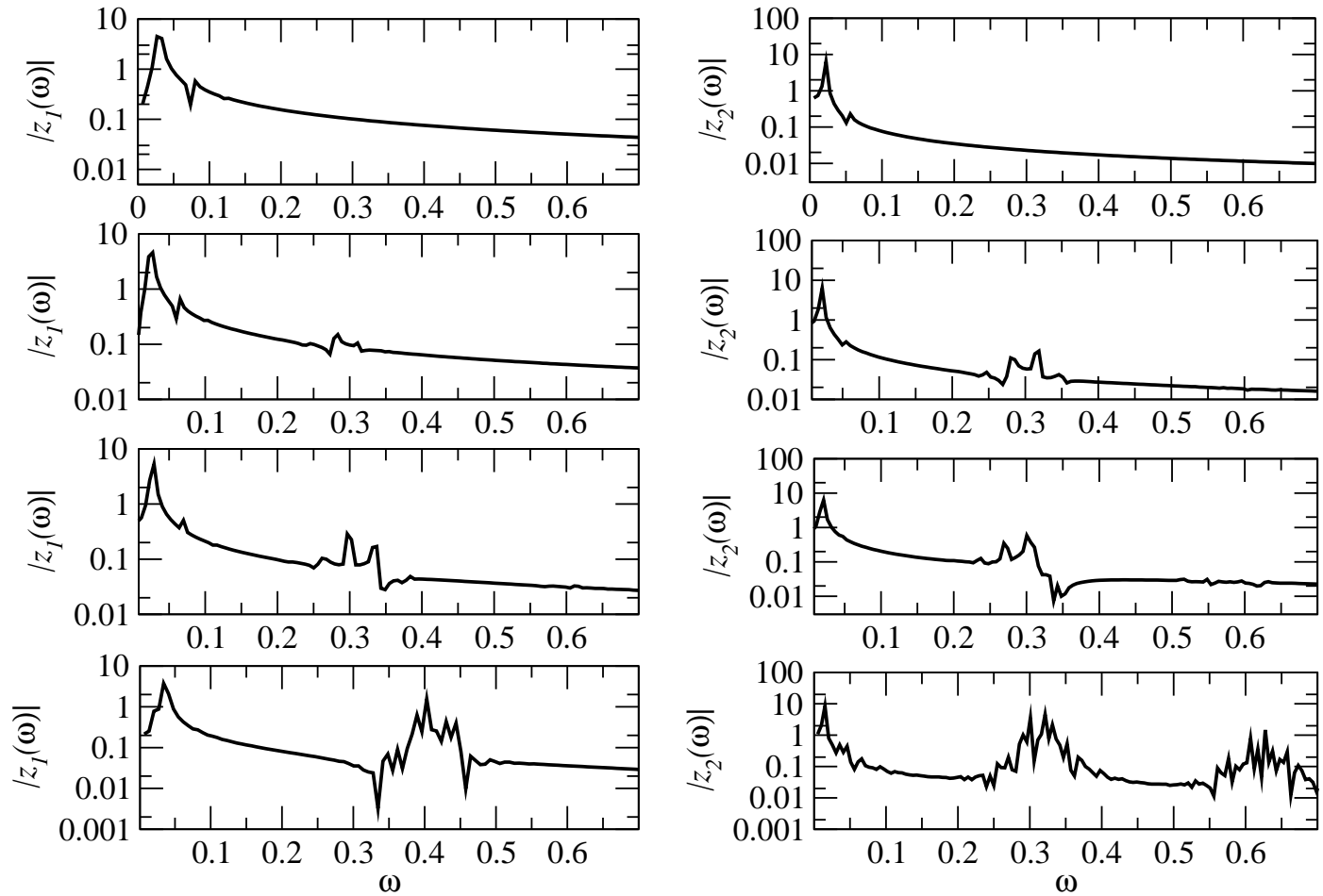

Figure 4. Josephson regime. The absolute value of $\left|z_{n}(\omega)\right|$ of the Fourier transform of the fractional imbalances vs. frequency $\omega$. Note that the vertical axis is in logarithmic scale. The parameters, initial conditions, and units as in Fig. 1.

\begin{tabular}{|c|c|c|c|c|}
\hline$\widetilde{\Omega}$ & $\bar{\omega}_{1}$ & $\bar{\omega}_{2}$ & $r_{1}$ & $r_{2}$ \\
\hline 0 & 0.0268 & 0.0224 & 0 & 0 \\
$K$ & 0.0272 & 0.0220 & 0.015 & 0.018 \\
$3 K$ & 0.0291 & 0.0211 & 0.086 & 0.060 \\
$20 K$ & 0.0341 & 0.0153 & 0.27 & 0.32 \\
\hline
\end{tabular}

Table 1. Spectra of Fig. 4. Frequencies $\bar{\omega}_{n}$ of the maxima of $\left|z_{n}(\omega)\right|$ for different values of $\tilde{\Omega}$ and their relative changes $r_{n}$ with respect to the absence of the Rabi coupling.

For the investigation of the self-trapped dynamics let us consider again Fig. 2 and also Figs. 5 and 6. In Fig. 5 we report the dynamics in the planes $\left(z_{n}, \theta\right)$, while in Fig. 6) we show the absolute value $\left|z_{n}(\omega)\right|$ of the Fourier transform of $z_{n}(t)$. From these figures, we see that in correspondence to high value of the linear coupling constant, an increasing complexity is observed within the dynamics supported by the junction.

It is important to stress that it exists a crossover value of the intra-species interaction, say $U_{S T}$, (note that we are setting $U_{1}=U_{2} \equiv U$ ), such that the two components are both self-trapped when $U>U_{S T}$. By integrating the ODEs (13)(14), we study $U_{S T}$ as a function of the absolute value of the inter-species interaction amplitude $U_{12}$ for different values of $\tilde{\Omega}$. The results of this study are presented in Fig. 7. ¿From the plots there reported, we can see that, once fixed $U_{12}$, the greater is $\tilde{\Omega}$, the greater is the value of the intra-species interaction necessary to achieve the self-trapping. 
Rabi-Josephson oscillations and self-trapped dynamics in atomic junctions with two bosonic species12
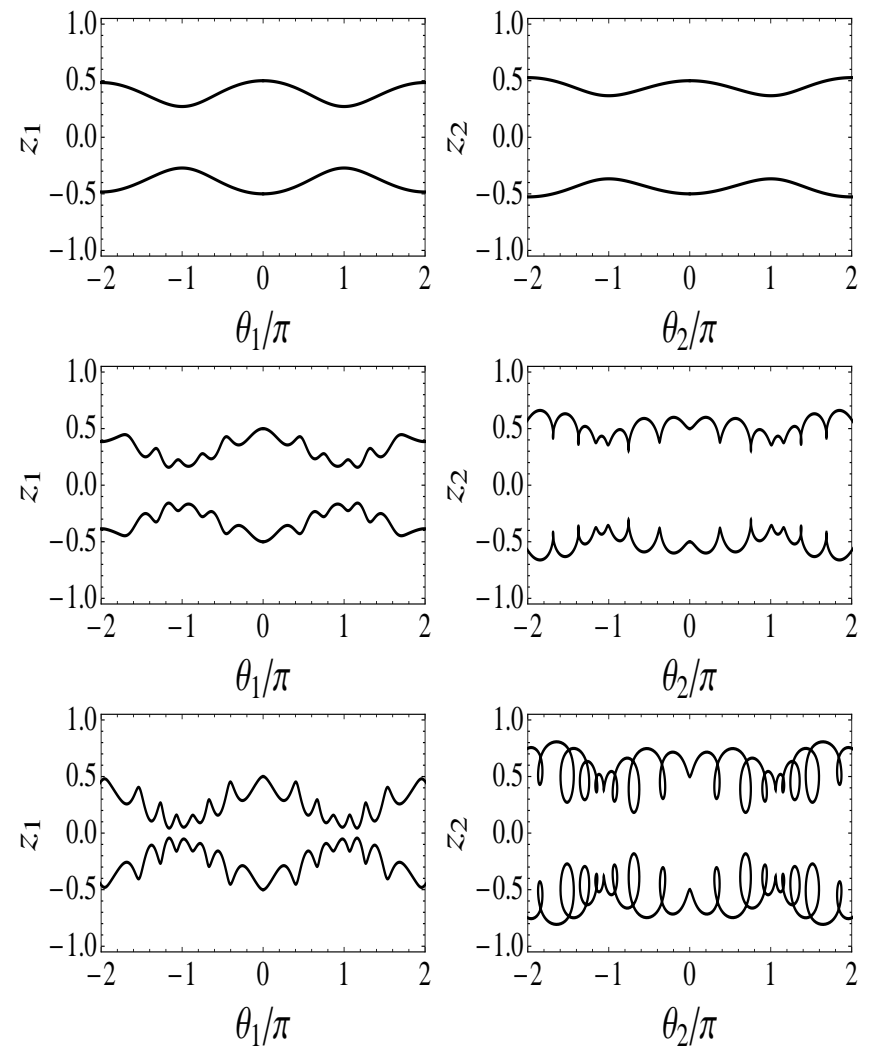

Figure 5. Self-trapping regime. The dynamics of the finite-dimensional system in the planes of fractional imbalances $z_{n}(t)$ and phases $\theta_{n}(t)$. The parameters, initial conditions, and units as in Fig. 2

\section{Conclusion}

In this work, we have introduced the model which allows one to study the interplay of the Josephson and Rabi oscillations in a binary Bose-Einstein condensate trapped in the double-well potential structure. The Rabi coupling is provided by the interconversion between the two species of the condensate, which represent distinct hyperfine states of the same atom.

To capture core features of the dynamics, we have derived a finite-mode approximation with four degrees of freedom, that represent the populations of the two species in the two symmetric potential wells. Comparison to full simulations of the underlying Gross-Pitaevskii system demonstrates that the truncated system provided for a reasonable accuracy. Systematic simulations of the system reveal the transition from regular Josephson oscillations to complex dynamics with the increase of the Rabi interconversion rate. Within the framework of this analysis, we have discussed the possibility to infer, at least at a qualitative level, the behavior of the fractional imbalances and the dynamics in the plane $z_{n}(t)-\theta_{n}(t)$ proceeding from the Fourier analysis of the fractional imbalances, especially for large Rabi couplings.

We have analyzed the dynamics of the atomic Josephson junction when both the components are self-trapped as well. We have shown that also in this case the truncated 
Rabi-Josephson oscillations and self-trapped dynamics in atomic junctions with two bosonic species13
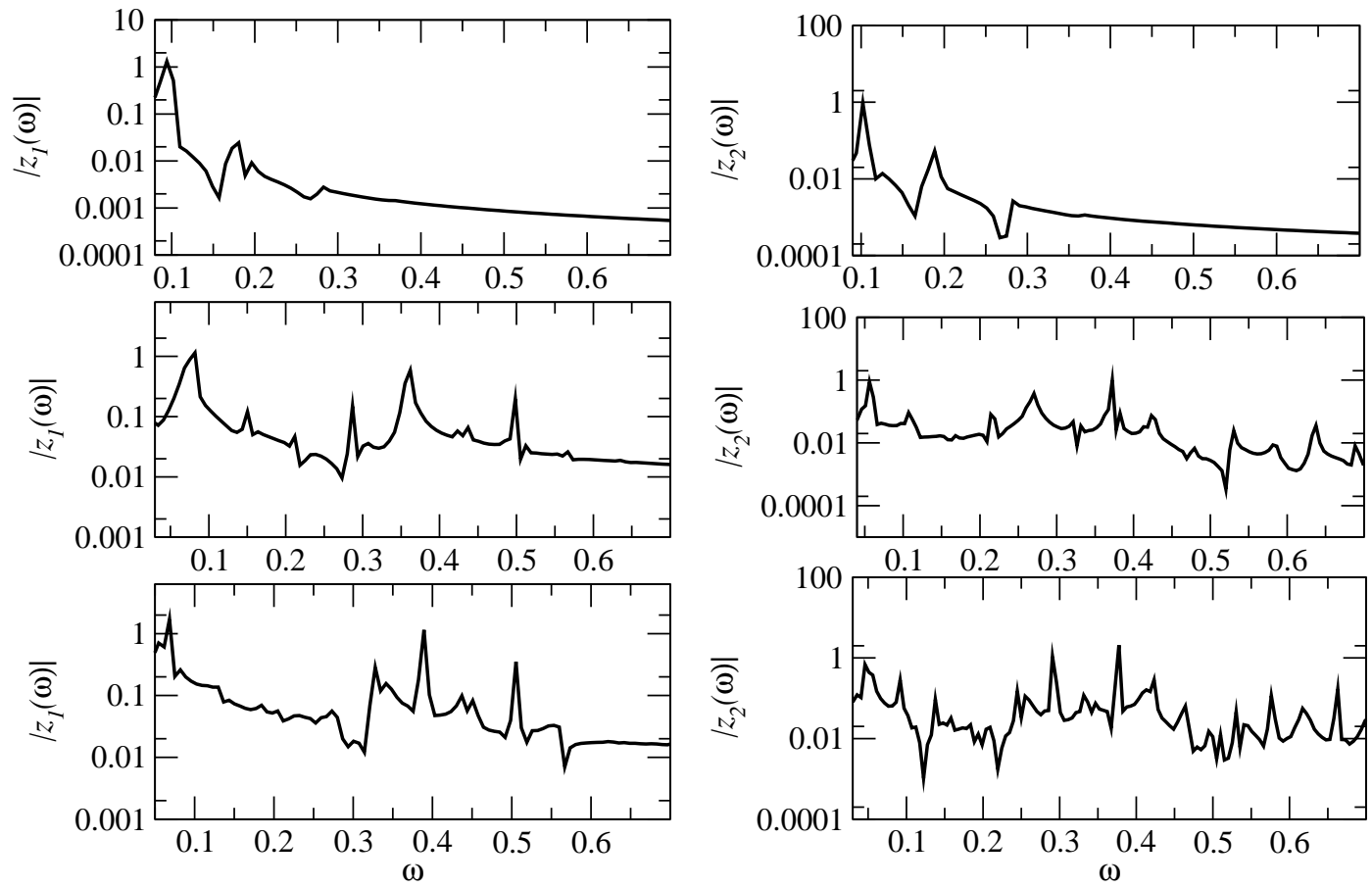

Figure 6. Self-trapping regime. The absolute value of $\left|z_{n}(\omega)\right|$ of the Fourier transform of the fractional imbalances vs. frequency $\omega$ in the self-trapping regime. Note that the vertical axis is in logarithmic scale. The parameters, initial conditions, and units as in Fig. 2 .

system gives rise to reliable predictions since the good agreement with the predictions of the associated Gross-Pitaevskii system. We have studied the influence of the linear coupling on the self-trapping onset.

\section{Appendix}

In this appendix, we discuss the path followed in deriving the evolution equations for the fractional imbalances $z_{n}=\left(N_{n}^{L}-N_{n}^{R}\right) / N_{n}$ and the intra-species relative phases $\theta_{n}=\theta_{n}^{R}-\theta_{n}^{L}$, i.e. Eq. (13) and the Eq. (14). We start by deriving the effective Lagrangian $L_{\text {eff }}$ in terms of variables $N_{n}^{\alpha}$ and $\theta_{n}^{\alpha}$ :

$$
\begin{aligned}
L_{\mathrm{eff}} & =\sum_{n=1,2}\left[-\hbar \dot{\theta}_{n}^{L} N_{n}^{L}-\hbar \dot{\theta}_{n}^{R} N_{n}^{R}-E_{n}^{L} N_{n}^{L}-E_{n}^{R} N_{n}^{R}\right. \\
& +2 K_{n} \sqrt{N_{n}^{L} N_{n}^{R}} \cos \left(\theta_{n}^{R}-\theta_{n}^{L}\right) \\
& -\left(\frac{U_{n}^{L}}{2}\left(N_{n}^{L}\right)^{2}+\frac{U_{n}^{R}}{2}\left(N_{n}^{R}\right)^{2}\right) \\
& -2 K_{c, n} N_{n} \sqrt{N_{n}^{L} N_{n}^{R}} \cos \left(\theta_{n}^{R}-\theta_{n}^{L}\right) \\
& -V_{n} N_{n}^{L} N_{n}^{R}\left(2+\cos 2\left(\theta_{n}^{R}-\theta_{n}^{L}\right)\right] \\
& -U_{12}^{L} N_{1}^{L} N_{2}^{L}-U_{12}^{R} N_{1}^{R} N_{2}^{R} \\
& -2 K_{c, 12}\left(\left(N_{1}^{L}+N_{1}^{R}\right) \sqrt{N_{2}^{L} N_{2}^{R}} \cos \left(\theta_{2}^{R}-\theta_{2}^{L}\right)\right.
\end{aligned}
$$


Rabi-Josephson oscillations and self-trapped dynamics in atomic junctions with two bosonic species14

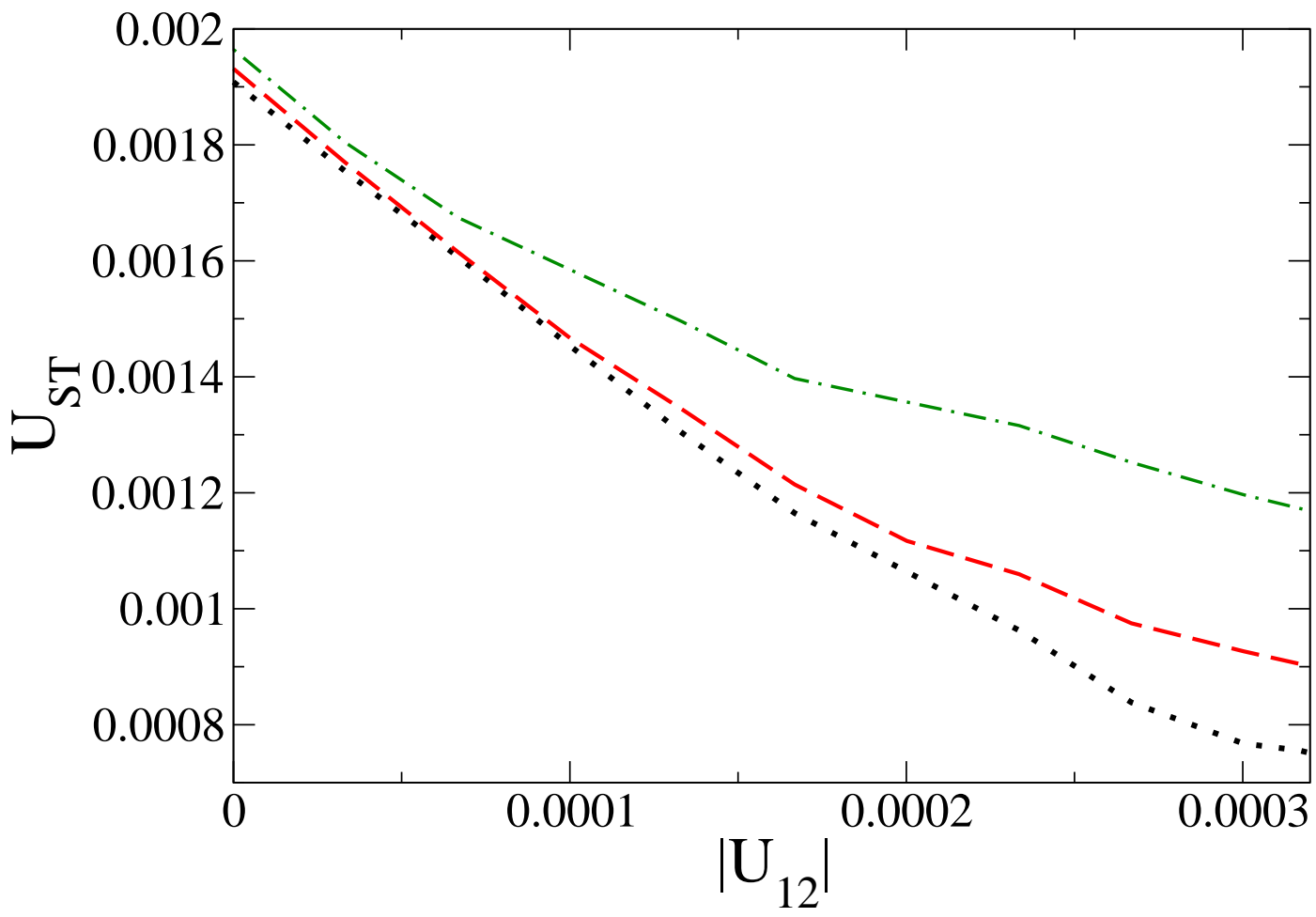

Figure 7. Self-trapping crossover value $U_{S T}$ as a function of the inter-species interaction $\left|U_{12}\right|$ for different values of $\tilde{\Omega}$. Dotted line: $\tilde{\Omega}=0$; dashed line: $\tilde{\Omega}=5 K$; dot-dashed line: $\tilde{\Omega}=10 \mathrm{~K}$. The double-well potential, initial conditions and units as in Fig. 2,

$$
\begin{aligned}
& \left.+\left(N_{2}^{L}+N_{2}^{R}\right) \sqrt{N_{1}^{L} N_{1}^{R}} \cos \left(\theta_{1}^{R}-\theta_{1}^{L}\right)\right) \\
& -V_{12}\left(N_{1}^{L} N_{2}^{R}+N_{1}^{R} N_{2}^{L}\right) \\
& -4 V_{12}\left(\sqrt{N_{1}^{L} N_{1}^{R}} \sqrt{N_{2}^{L} N_{2}^{R}} \cos \left(\theta_{1}^{R}-\theta_{1}^{L}\right) \cos \left(\theta_{2}^{R}-\theta_{2}^{L}\right)\right) \\
& -2\left(R_{12}^{L} \sqrt{N_{1}^{L} N_{2}^{L}} \cos \left(\theta_{2}^{L}-\theta_{1}^{L}\right)\right) \\
& -2\left(R_{12}^{R} \sqrt{N_{1}^{R} N_{2}^{R}} \cos \left(\theta_{2}^{R}-\theta_{1}^{R}\right)\right) \\
& -2\left(S_{12}^{L R} \sqrt{N_{1}^{L} N_{2}^{R}} \cos \left(\theta_{2}^{R}-\theta_{1}^{L}\right)\right) \\
& -2\left(T_{12}^{R L} \sqrt{N_{1}^{R} N_{2}^{L}} \cos \left(\theta_{2}^{L}-\theta_{1}^{R}\right)\right)
\end{aligned}
$$

where the following constants are introduced:

$$
\begin{aligned}
& E_{n}^{\alpha}=\int d z\left[\frac{\hbar^{2}}{2 m}\left(\frac{d \phi_{n}^{\alpha}}{d z}\right)^{2}+\right. \\
& \left.\left(\frac{\hbar^{2}}{2 m a_{\perp, n}^{2}}+\frac{m \omega_{n}^{2} a_{\perp, n}^{2}}{2}+V_{\mathrm{DWP}}(z)+\frac{(-1)^{n} \hbar \delta}{2}\right)\left(\phi_{n}^{\alpha}\right)^{2}\right] \\
& U_{n}^{\alpha}=\tilde{g}_{n} \int d z\left(\phi_{n}^{\alpha}\right)^{4}
\end{aligned}
$$


Rabi-Josephson oscillations and self-trapped dynamics in atomic junctions with two bosonic species15

$$
\begin{aligned}
& K_{n}=-\int d z\left[\frac{\hbar^{2}}{2 m} \frac{d \phi_{n}^{L}}{d z} \frac{d \phi_{n}^{R}}{d z}+V_{\mathrm{DWP}}(z) \phi_{n}^{L} \phi_{n}^{R}\right] \\
& K_{c, n}=\tilde{g}_{n} \int d z\left(\phi_{n}^{\alpha}(z)\right)^{3} \phi_{n}^{\beta}(z) \\
& V_{n}=2 \tilde{g}_{n} \int d z\left(\phi_{n}^{\alpha}(z)\right)^{2}\left(\phi_{n}^{\beta}(z)\right)^{2} \\
& U_{12}^{\alpha}=\tilde{g}_{12} \int d z\left(\phi_{1}^{\alpha}\right)^{2}\left(\phi_{2}^{\alpha}\right)^{2} \\
& K_{c, 12}=\tilde{g}_{12} \int d z\left(\phi_{1}^{\alpha}(z)\right)^{3} \phi_{2}^{\beta}(z) \\
& V_{12}=\tilde{g}_{12} \int d z\left(\phi_{1}^{\alpha}(z)\right)^{2}\left(\phi_{2}^{\beta}(z)\right)^{2} \\
& R_{12}^{\alpha}=(\tilde{\Omega} / 2) \int d z \phi_{1}^{\alpha}(z) \phi_{2}^{\alpha}(z) \\
& S_{12}^{L R}=(\tilde{\Omega} / 2) \int d z \phi_{1}^{L}(z) \phi_{2}^{R}(z) \\
& T_{12}^{R L}=(\tilde{\Omega} / 2) \int d z \phi_{1}^{R}(z) \phi_{2}^{L}(z) .
\end{aligned}
$$

To analyze the finite-mode dynamics induced by Lagrangian (19), we define the canonical momenta conjugate to generalized coordinates $\hbar \theta_{n}^{\alpha}$ :

$$
p_{\theta_{n}^{\alpha}}=\frac{1}{\hbar} \frac{\partial L_{\mathrm{eff}}}{\partial \dot{\theta}_{n}^{\alpha}}=-N_{n}^{\alpha} .
$$

Accordingly, the Hamiltonian of the system is written in terms of the canonical coordinates and momenta as follows:

$$
\begin{aligned}
H & =-\sum_{n=1,2}\left[p_{\theta_{n}^{L}} E_{n}^{L}+p_{\theta_{n}^{R}} E_{n}^{R}\right] \\
& -\sum_{n=1,2} 2 K_{n}^{\alpha} \sqrt{p_{\theta_{n}^{L}} p_{\theta_{n}^{R}}} \cos \left(\theta_{n}^{L}-\theta_{n}^{R}\right) \\
& +\sum_{n=1,2}\left[\frac{U_{n}^{L}}{2} p_{\theta_{n}^{L}}^{2}+\frac{U_{n}^{R}}{2} p_{\theta_{n}^{R}}^{2}\right. \\
& -2 K_{c, n}\left(p_{\theta_{n}^{R}}+p_{\theta_{n}^{L}}\right) \sqrt{p_{\theta_{n}^{R} p_{\theta_{n}^{L}}}} \cos \left(\theta_{n}^{R}-\theta_{n}^{L}\right) \\
& \left.+V_{n}\left(2+\cos 2\left(\theta_{n}^{L}-\theta_{n}^{R}\right)\right) p_{\theta_{n}^{L}} p_{\theta_{n}^{R}}\right] \\
& +U_{12}^{L} p_{\theta_{1}^{L}} p_{\theta_{2}^{L}}+U_{12}^{R} p_{\theta_{1}^{R}} p_{\theta_{2}^{R}} \\
& -2 K_{c, 12}\left[\left(p_{\theta_{1}^{L}}+p_{\theta_{1}^{R}}\right) \sqrt{p_{\theta_{2}^{L}} p_{\theta_{2}^{R}}} \cos \left(\theta_{2}^{R}-\theta_{2}^{L}\right)\right. \\
& \left.+\left(p_{\theta_{2}^{L}}+p_{\theta_{2}^{R}}\right) \sqrt{p_{\theta_{1}^{L}} p_{\theta_{1}^{R}}} \cos \left(\theta_{1}^{R}-\theta_{1}^{L}\right)\right] \\
& +V_{12}\left(p_{\theta_{1}^{L}} p_{\theta_{2}^{R}}+p_{\theta_{1}^{R}} p_{\theta_{2}^{L}}\right) \\
& +4 V_{12}\left(\sqrt{p_{\theta_{1}^{L}} p_{\theta_{1}^{R}}} \sqrt{p_{\theta_{2}^{L}} p_{\theta_{2}^{R}}} \cos \left(\theta_{1}^{R}-\theta_{1}^{L}\right) \cos \left(\theta_{2}^{R}-\theta_{2}^{L}\right)\right) \\
& +2\left(R_{12}^{L} \sqrt{p_{\theta_{1}^{L}} p_{\theta_{2}^{L}}} \cos \left(\theta_{2}^{L}-\theta_{1}^{L}\right)\right) \\
& +2\left(R_{12}^{R} \sqrt{p_{\theta_{1}^{R}} p_{\theta_{2}^{R}}} \cos \left(\theta_{2}^{R}-\theta_{1}^{R}\right)\right)
\end{aligned}
$$


Rabi-Josephson oscillations and self-trapped dynamics in atomic junctions with two bosonic species16

$$
\begin{aligned}
& +2\left(S_{12}^{L R} \sqrt{p_{\theta_{1}^{L}} p_{\theta_{2}^{R}}} \cos \left(\theta_{2}^{R}-\theta_{1}^{L}\right)\right) \\
& +2\left(T_{12}^{R L} \sqrt{p_{\theta_{1}^{R}} p_{\theta_{2}^{L}}^{L}} \cos \left(\theta_{2}^{L}-\theta_{1}^{R}\right)\right) .
\end{aligned}
$$

The evolution equations for populations $N_{n}^{\alpha}$ and phases $\theta_{n}^{\alpha}$ are derived, as the canonical equations, from the Hamiltonian: (22)

$$
\dot{p}_{\theta_{n}^{\alpha}}=-\frac{1}{\hbar} \frac{\partial H}{\partial \theta_{n}^{\alpha}}, \dot{\theta}_{n}^{\alpha}=\frac{1}{\hbar} \frac{\partial H}{\partial p_{\theta_{n}^{\alpha}}} .
$$

We observe that due to the orthonormality of the decomposition basis, $R_{12}^{\alpha}=\tilde{\Omega} / 2$ and $S_{12}^{L R}=T_{21}^{R L}=0$. From the symmetry between the two wells in the DWP structure it also follows that $E_{n}^{L}=E_{n}^{R} \equiv E_{n}, U_{n}^{L}=U_{n}^{R} \equiv U_{n}, U_{12}^{L}=U_{12}^{R} \equiv U_{12}$. Using Eq. (23), we derive explicit evolution equations for the Eq. (13) and the evolution equations for the total numbers of particles of each component $N_{n}$ and the respective phases, $\gamma_{\alpha}=\theta_{1}^{\alpha}-\theta_{2}^{\alpha}$ (recall that $\alpha=L, R)$ :

$$
\begin{aligned}
\dot{z}_{n}= & -\frac{2\left(K_{n}-K_{c, i} N_{n}\right)}{\hbar} \sqrt{1-z_{n}^{2}} \sin \theta_{n}+\frac{V_{n} N_{n}}{2 \hbar}\left(1-z_{n}^{2}\right) \sin 2 \theta_{n} \\
+ & \frac{2}{\hbar}\left(V_{12} \sqrt{1-z_{3-n}^{2}} \cos \theta_{3-n}+K_{c, 12}\right) N_{3-n} \sqrt{1-z_{n}^{2}} \sin \theta_{n} \\
& \mp \frac{\tilde{\Omega}}{2 \hbar} \sqrt{N_{1} N_{2}}\left(\sqrt{\left(1+z_{1}\right)\left(1+z_{2}\right)} \sin \gamma_{L}-\sqrt{\left(1-z_{1}\right)\left(1-z_{2}\right)} \sin \gamma_{R}\right), \\
\dot{\theta}_{n}= & \frac{U_{n}-V_{n}}{\hbar} N_{n} z_{n}+\frac{2\left(K_{n}-K_{c, n} N_{n}\right)}{\hbar} \frac{z_{n} \cos \theta_{n}}{\sqrt{1-z_{n}^{2}}} \\
- & \frac{V_{n} N_{n}}{2 \hbar} z_{n} \cos 2 \theta_{n}+\frac{U_{12}-V_{12}}{\hbar} N_{3-n} z_{3-n} \\
- & \frac{2}{\hbar}\left[V_{12} \sqrt{1-z_{3-n}^{2}} \cos \theta_{3-n}+K_{c, 12}\right] N_{3-n} \frac{z_{n} \cos \theta_{n}}{\sqrt{1-z_{n}^{2}}} \\
+ & \frac{\tilde{\Omega}}{2 \hbar} \sqrt{\frac{N_{3-n}}{N_{n}}}\left(\sqrt{\frac{1+z_{3-n}}{1+z_{n}}} \cos \gamma_{L}-\sqrt{\frac{1-z_{3-n}}{1-z_{n}}} \cos \gamma_{R}\right) \\
& \dot{N}_{n}= \pm\left(-\frac{\tilde{\Omega}}{2 \hbar} \sqrt{N_{1} N_{2}}\right)\left(\sqrt{\left(1+z_{1}\right)\left(1+z_{2}\right)} \sin \gamma_{L}+\sqrt{\left(1-z_{1}\right)\left(1-z_{2}\right)} \sin \gamma_{R}\right), \\
& \dot{\gamma}_{L}=\frac{1}{2 \hbar}\left(N_{1}\left(U_{12}-U\right)\left(1+z_{1}\right)-N_{2}\left(U_{12}-U\right)\left(1+z_{2}\right)+\Delta E\right) \\
+ & \frac{1}{\hbar}\left(K_{1} \sqrt{\frac{1-z_{1}}{1+z_{1}}} \cos \theta_{1}-K_{2} \sqrt{\frac{1-z_{2}}{1+z_{2}}} \cos \theta_{2}\right) \\
- & \frac{\tilde{\Omega}}{2 \hbar \sqrt{N_{1} N_{2}}}\left(\frac{N_{2}\left(1+z_{2}\right)-N_{1}\left(1+z_{1}\right)}{\sqrt{\left(1+z_{1}\right)\left(1+z_{2}\right)}}\right) \cos \gamma_{L} \\
+ & \frac{K_{c, 12}}{\hbar}\left[N_{1} \sqrt{1-z_{1}^{2}} \cos \theta_{1}-N_{2} \sqrt{1-z_{2}^{2}} \cos \theta_{2}\right]- \\
& \frac{V}{2 \hbar}\left[N_{1}\left(1-z_{1}\right)\left(2+\cos 2 \theta_{1}\right)-N_{2}\left(1-z_{2}\right)\left(2+\cos 2 \theta_{2}\right)\right]- \\
& \frac{1}{\hbar}\left[K_{c, 1} N_{1}\left(2+z_{1}\right)+K_{c, 12} N_{2}+V_{12} N_{2} \sqrt{1-z_{2}^{2}} \cos \theta_{2}\right] \sqrt{\frac{1-z_{1}}{1+z_{1}}} \cos \theta_{1}+ \\
&
\end{aligned}
$$


Rabi-Josephson oscillations and self-trapped dynamics in atomic junctions with two bosonic species17

$$
\begin{aligned}
& \frac{1}{\hbar}\left[K_{c, 2} N_{2}\left(2+z_{2}\right)+K_{c, 12} N_{1}\right. \\
+ & \left.V_{12} N_{1} \sqrt{1-z_{1}^{2}} \cos \theta_{1}\right] \sqrt{\frac{1-z_{2}}{1+z_{2}}} \cos \theta_{2}+\frac{V_{12}}{2}\left(N_{1}\left(1-z_{1}\right)-N_{2}\left(1-z_{2}\right)\right), \\
& \dot{\gamma}_{R}=\frac{1}{2 \hbar}\left(N_{1}\left(U_{12}-U\right)\left(1-z_{1}\right)-N_{2}\left(U_{12}-U\right)\left(1-z_{2}\right)+\Delta E\right) \\
+ & \frac{1}{\hbar}\left(K_{1} \sqrt{\frac{1+z_{1}}{1-z_{1}}} \cos \theta_{1}-K_{2} \sqrt{\frac{1+z_{2}}{1-z_{2}}} \cos \theta_{2}\right)- \\
& \frac{\tilde{\Omega}}{\hbar \sqrt{N_{1} N_{2}}}\left(\frac{N_{2}\left(1-z_{2}\right)-N_{1}\left(1-z_{1}\right)}{\sqrt{\left(1-z_{1}\right)\left(1-z_{2}\right)}}\right) \cos \gamma_{R} \\
+ & \frac{K_{c, 12}}{\hbar}\left[N_{1} \sqrt{1-z_{1}^{2}} \cos \theta_{1}-N_{2} \sqrt{1-z_{2}^{2}} \cos \theta_{2}\right]- \\
& \frac{V}{2 \hbar}\left[N_{1}\left(1+z_{1}\right)\left(2+\cos 2 \theta_{1}\right)-N_{2}\left(1+z_{2}\right)\left(2+\cos 2 \theta_{2}\right)\right]- \\
& \frac{1}{\hbar}\left[K_{c, 1} N_{1}\left(2-z_{1}\right)+K_{c, 12} N_{2}+V_{12} N_{2} \sqrt{1-z_{2}^{2}} \cos \theta_{2}\right] \sqrt{\frac{1+z_{1}}{1-z_{1}}} \cos \theta_{1}+ \\
& \frac{1}{\hbar}\left[K_{c, 2} N_{2}\left(2-z_{2}\right)+K_{c, 12} N_{1}\right. \\
+ & \left.V_{12} N_{1} \sqrt{1-z_{1}^{2}} \cos \theta_{1}\right] \sqrt{\frac{1+z_{2}}{1-z_{2}}} \cos \theta_{2}+\frac{V_{12}}{2}\left(N_{1}\left(1+z_{1}\right)-N_{2}\left(1+z_{2}\right)\right),
\end{aligned}
$$

[1] A. Smerzi, S. Fantoni, S. Giovanazzi, and S. R. Shenoy, Phys. Rev. Lett. 79, 4950 (1997); S. Raghavan, A. Smerzi, S. Fantoni, and S. R. Shenoy, Phys. Rev. A 59, 620 (1999); S. Narayana and Y. Sato, Phys. Rev. Lett. 105, 205302 (2010).

[2] C. J. Myatt, E. A. Burt, R. W. Ghrist, E. A. Cornell, and C. E. Wieman, Phys. Rev. Lett 78, 586 (1997).

[3] S. Ashhab and C. Lobo, Phys. Rev. A 66, 013609 (2002).

[4] X. Xu, L. Lu, Y. Li, Phys. Rev. A 78, 043609 (2008).

[5] I. I. Satija, P. Naudus, R. Balakrishnan, J. Heward, M. Edwards, C. W. Clark, Phys. Rev. A 79, 033616 (2009).

[6] B. Julia-Diaz, M. Guilleumas, M. Lewenstein, A. Polls, A. Sanpera, Phys. Rev. A 80, 023616 (2009).

[7] G. Mazzarella, M. Moratti, L. Salasnich, M. Salerno and F. Toigo, J. Phys. B: At. Mol. Opt. Phys. 42, 125301 (2009).

[8] G. Mazzarella, M. Moratti, L. Salasnich, and F. Toigo, J. Phys. B: At. Mol. Opt. Phys. 43, 065303 (2010).

[9] B. Julia-Diaz, M. Mele-Messeguer, M. Guilleumas, and A. Polls, Phys. Rev. A 80, 043622 (2009).

[10] C. Wang, P. G. Kevrekidis, N. Whitaker and B. A. Malomed, Physica D 327, 2922 (2008).

[11] R. J. Ballagh, K. Burnett, and T. F. Scott, Phys. Rev. Lett. 78, 1607 (1997).

[12] J. Williams, R. Walser, J. Cooper, E. Cornell, and M. Holland, Phys. Rev. A 59, R31 (1999).

[13] P. Öhberg and S. Stenholm, Phys. Rev. A 59, 3890 (1999); S. D. Jenkins and T. A. B. Kennedy, Phys. Rev. A 68, 053607 (2003).

[14] M. Yasunaga and M. Tsubota, J. Low Temp. Phys., published online: doi 10.1007/s10909-0099942-7.

[15] M. Tujillo-Martinez, A. Posazhennikova, and J. Kroha, Phys. Rev. Lett. 103, 105302 (2009).

[16] L. Pezzé , A. Smerzi, G. P. Berman, A. R. Bishop, and L. A. Collins, Phys. Rev. A 74, 033610 (2006). 
[17] B. Xia, W. Hai and G. Chong, Phys. Lett. A 351, 136 (2006).

[18] T. Zibold, E. Nicklas, C. Gross, M.K. Oberthaler, Phys. Rev. Lett. 105, 204101 (2010).

[19] K. Marzlin, W. Zhang, and Wright, Phys. Rev. Lett. 79, 4728 (1997); R. Dum, J. I. Cirac, M. Lewenstein, and P. Zoller, Phys. Rev. Lett. 80, 2972 (1998).

[20] P. Zhang, P. Naidon, and M. Ueda, Phys. Rev. Lett. 103, 133202 (2009).

[21] L. Salasnich, A. Parola, and L. Reatto, Phys. Rev. A 65, 043614 (2002); L. Salasnich, A. Parola, and L. Reatto, Phys. Rev. A 70, 013606 (2004); L. Salasnich and B. A. Malomed, Phys. Rev. A 74, 053610 (2006).

[22] C. J. Milburn, J. Corney, E. M. Wright, and D. F. Walls, Phys. Rev. A 55, 4318 (1997).

[23] L. Landau and L. Lifshitz, Course in Theoretical Physics, Vol. 3, Quantum Mechanics: NonRelativistic Theory, (Pergamon, New York, 1959). 